\title{
The Security of Abreast-DM in the Ideal Cipher Model
}

\author{
Jooyoung Lee, Daesung Kwon \\ The Attached Institute of Electronics and Telecommunications Research Institute \\ Yuseong-gu, Daejeon, Korea 305-390 \\ jlee05@ensec.re.kr,ds_kwon@ensec.re.kr
}

\begin{abstract}
In this paper, we give a security proof for ABREAST-DM in terms of collision resistance and preimage resistance. As old as TANDEM-DM, the compression function ABREAST-DM is one of the most well-known constructions for double block length compression functions. The bounds on the number of queries for collision resistance and preimage resistance are given by $O\left(2^{n}\right)$. Based on a novel technique using query-response cycles, our security proof is simpler than those for MDC-2 and TANDEM-DM. We also present a wide class of ABREAST-DM variants that enjoy a birthday-type security guarantee with a simple proof.
\end{abstract}

\section{Introduction}

A cryptographic hash function takes a message of arbitrary length, and returns a bit string of fixed length. The most common way of hashing variable length messages is to iterate a fixed-size compression function according to the Merkle-Damgård paradigm. The underlying compression function can either be constructed from scratch, or be built upon off-the-shelf cryptographic primitives such as blockciphers. Recently, the blockcipher-based construction is attracting renewed interest, as many dedicated hash functions, including those most common in practical applications, exhibit serious security weaknesses $[1,6,14,15,20,24-26]$. Conveniently choosing an extensively studied blockcipher in the blockcipher-based construction, one can easily transfer the trust in the existing algorithm to the hash function. This approach is particularly useful in highly constrained environments such as RFID systems, since a single implementation of a blockcipher can be used for both a blockcipher and a hash function. Compared to blockciphers, the most dedicated hash functions require significant amounts of state and the operations in their designs are not hardware friendly [3].

Compression functions based on blockciphers have been widely studied $[2,4,8-11,13,16-19$, 21-23]. The most common approach is to construct a $2 n$-to- $n$ bit compression function using a single call to an $n$-bit blockcipher. However, such a function, called a single block length (SBL) compression function, might be vulnerable to collision attacks due to its short output length. For example, one could successfully mount a birthday attack on a compression function based on AES128 using approximately $2^{64}$ queries. This observation motivated substantial research on double block length (DBL) compression functions, where the output length is twice the block length of the underlying blockciphers.

Unfortunately, it turned out that a wide class of DBL compression functions of rate 1 are not optimally secure in terms of collision resistance and preimage resistance $[8,9,12]$. The most classical DBL compression functions of rate less than 1 include MDC-2, MDC-4, TANDEM-DM and Abreast-DM [5,13]. In 2007, 20 years after its original proposal, Steinberger first proved the collision resistance of MDC-2 in the ideal cipher model [23]. The author showed that an adversary asking less than $2^{3 n / 5}$ queries has only a negligible chance of finding a collision. Motivated by this work, Fleischmann et. al. proved the security of TANDEM-DM [7]. Similar to MDC-2, the security of TANDEM-DM is estimated in terms of a parameter, say, $\alpha$. Optimizing the parameter, they proved the collision resistance of TANDEM-DM up to the birthday bound. Currently, TANDEMDM and the Hirose's scheme [11] are the only rate 1/2 DBL compression functions that are known to have a birthday-type security guarantee. 
Results We give a security proof for ABREAST-DM in terms of collision resistance and preimage resistance. As old as TANDEM-DM, the compression function ABREAST-DM is known to be more advantageous than TANDEM-DM in that two encryptions involved can be computed in parallel. The bounds on the number of queries for collision resistance and preimage resistance are given by $O\left(2^{n}\right)$. Our security proof using certain cyclic structures, called query-response cycles, is much simpler than those for MDC-2 and TANDEM-DM. The query-response cycle technique also allows us to present a wide class of ABREAST-DM variants that enjoy a birthday-type security guarantee with a simple proof. It is shown that this class includes the Hirose's scheme [11] as a special case. We note, however, this technique does not apply directly to MDC-2 and TANDEM-DM, since two encryptions in these compression functions are computed in serial and hence it is infeasible to define query-response cycles. The underlying blockcipher of ABREAsT-DM use $2 n$-bit keys, while MDC-2 accepts $n$-bit keys. For this reason, it seems to be natural that the security proof of MDC-2 is more challenging.

\section{Preliminaries}

General Notations For a positive integer $n$, we let $I_{n}=\{0,1\}^{n}$ denote the set of all bitstrings of length $n$. For two bitstrings $A$ and $B, A \mid B$ and $\bar{A}$ denote the concatenation of $A$ and $B$, and the bitwise complement of $A$, respectively. For a set $U$, we write $u \stackrel{\$}{\leftarrow} U$ to denote uniform random sampling from the set $U$ and assignment to $u$.

Ideal Cipher Model For positive integers $n$ and $k$, let

$$
B C(n, k)=\left\{E: I_{n} \times I_{k} \rightarrow I_{n}: \forall K \in I_{k}, E(\cdot, K) \text { is a permutation on } I_{n}\right\} .
$$

In the ideal cipher model, an $(n, k)$-blockcipher $E$ is chosen from $B C(n, k)$ uniformly at random. It allows for two types of oracle queries $E(X, K)$ and $E^{-1}(Y, K)$ for $X, Y \in I_{n}$ and $K \in I_{k}$. Here, $X, Y$ and $K$ are called a plaintext, a ciphertext and a key, respectively. The response to an inverse query $E^{-1}(Y, K)$ is $X \in I_{n}$ such that $E(X, K)=Y$.

The Abreast-DM Compression Function In the ideal cipher model, the ABREAST-DM compression function

$$
F^{A B R}: I_{n}^{3} \longrightarrow I_{n}^{2}
$$

has oracle access to an ideal cipher $E \in B C(n, 2 n)$, and computes $F^{A B R}\left(A_{1}, A_{2}, A_{3}\right),\left(A_{1}, A_{2}, A_{3}\right) \in$ $I_{n}^{3}$, by the algorithm described in Figure 1.
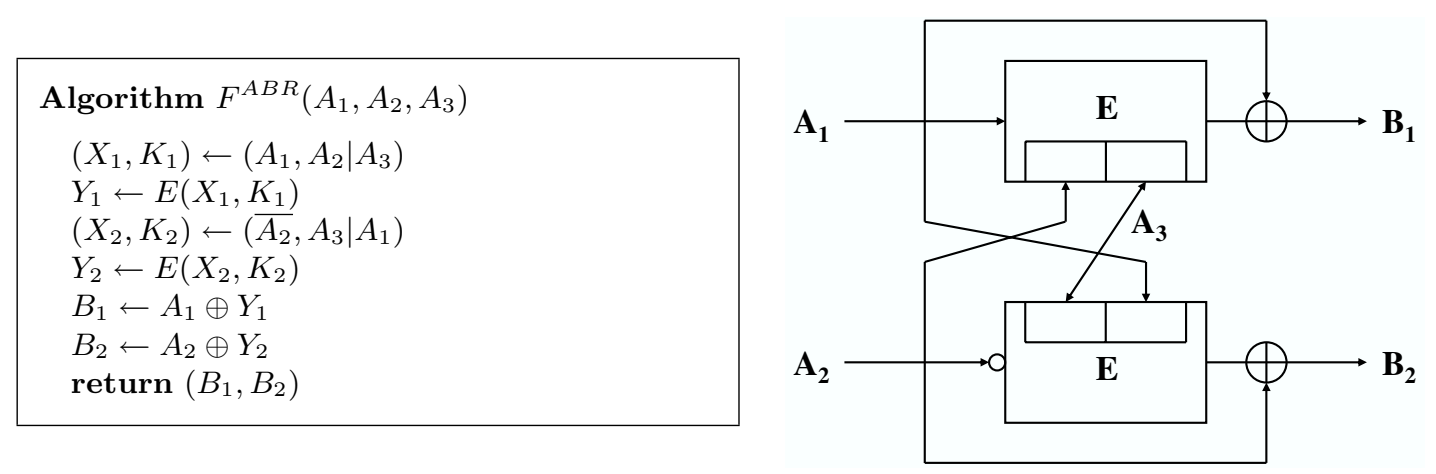

Fig. 1. The Abreast-DM compression function 
Collision Resistance and Preimage Resistance Let $F:=F^{A B R}$ be the ABREAST-DM compression function based on an ideal blockcipher $E \in B C(n, 2 n)$, and let $\mathcal{A}$ be an informationtheoretic adversary with oracle access to $E$ and $E^{-1}$. Then we execute the experiment $\operatorname{Exp}_{\mathcal{A}}^{\text {coll }}$ described in Figure 2(a), in order to quantify the collision resistance of $F$. The experiment records the queries that the adversary $\mathcal{A}$ makes into a query history $\mathcal{Q}$. A pair $(X, K, Y)$ is in the query history if $\mathcal{A}$ asks for $E(X, K)$ and gets back $Y$, or it asks for $E^{-1}(Y, K)$ and gets back $X$. For $A=\left(A_{1}, A_{2}, A_{3}\right) \in I_{n}^{3}$ and $B=\left(B_{1}, B_{2}\right) \in I_{n}^{2}$, we write

$$
A \vdash_{\mathcal{Q}} B
$$

if there exist query-response pairs $\left(X_{1}, K_{1}, Y_{1}\right),\left(X_{2}, K_{2}, Y_{2}\right) \in \mathcal{Q}$, satisfying the following equations.

$$
\begin{aligned}
\left(X_{1}, K_{1}\right) & =\left(A_{1}, A_{2} \mid A_{3}\right), \\
\left(X_{2}, K_{2}\right) & =\left(\overline{A_{2}}, A_{3} \mid A_{1}\right), \\
B_{1} & =A_{1} \oplus Y_{1}, \\
B_{2} & =A_{2} \oplus Y_{2} .
\end{aligned}
$$

Informally, $A \vdash_{\mathcal{Q}} B$ means that the query history $\mathcal{Q}$ determines the evaluation $F: A \mapsto B$. Now the collision-finding advantage of $\mathcal{A}$ is defined to be

$$
\operatorname{Adv}_{F}^{\text {coll }}(\mathcal{A})=\operatorname{Pr}\left[\operatorname{Exp}_{\mathcal{A}}^{\text {coll }}=1\right] .
$$

The probability is taken over the random blockcipher $E$ and $\mathcal{A}$ 's coins (if any). For $q>0$, we define $\mathbf{A d v}_{F}^{\text {coll }}(q)$ as the maximum of $\mathbf{A d v}_{F}^{\text {coll }}(\mathcal{A})$ over all adversaries $\mathcal{A}$ making at most $q$ queries.

The preimage resistance of $F$ is quantified similarly using the experiment $\operatorname{Exp}_{\mathcal{A}}^{\text {pre }}$ described in Figure 2(b). The adversary $\mathcal{A}$ chooses a single target image $B \in I_{n}^{2}$ before it begins making queries to $E^{ \pm 1}$. The preimage-finding advantage of $\mathcal{A}$ is defined to be

$$
\operatorname{Adv}_{F}^{\text {pre }}(\mathcal{A})=\operatorname{Pr}\left[\operatorname{Exp}_{\mathcal{A}}^{\text {pre }}=1\right] .
$$

For $q>0, \operatorname{Adv}_{F}^{\text {pre }}(q)$ is the maximum of $\operatorname{Adv}_{F}^{\text {pre }}(\mathcal{A})$ over all adversaries $\mathcal{A}$ making at most $q$ queries. The security definitions given in this section can be extended easily to any compression function built upon ideal primitives by appropriately defining the relation " $\vdash_{\mathcal{Q}}$ ".

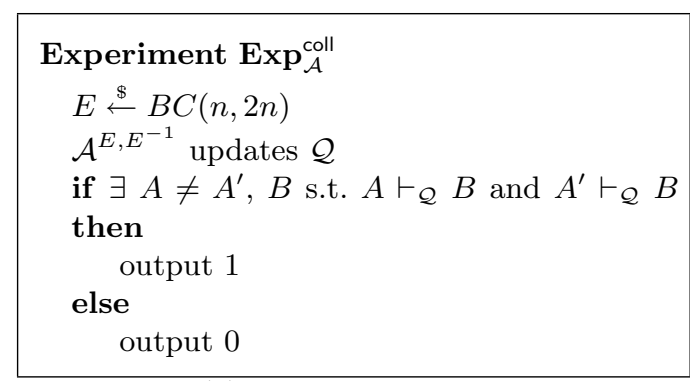

(a) Collision resistance

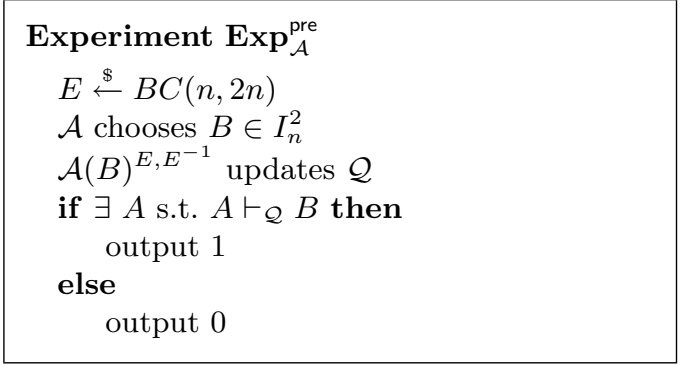

(b) Preimage resistance

Fig. 2. Experiments for quantification of collision resistance and preimage resistance

\section{Security of Abreast-DM}

\subsection{Query-response Cycle and Modified Adversary}

Let $F:=F^{A B R}$ be the ABREAST-DM compression function based on a blockcipher $E \in B C(n, 2 n)$, and let $\mathcal{Q}$ be the query history obtained by oracle access to $E$ and $E^{-1}$. Now we associate the 
query history $\mathcal{Q}$ with a direct graph $\mathcal{G}$ on $\mathcal{Q}$, where $\overrightarrow{Q Q^{\prime}} \in \mathcal{G}$ if and only if $Q=\left(A_{1}, A_{2} \mid A_{3}, Y_{1}\right)$ and $Q^{\prime}=\left(\overline{A_{2}}, A_{3} \mid A_{1}, Y_{2}\right)$ for some $A_{s}$ 's and $Y_{t}^{\prime}$ 's. A (direct) cycle in $\mathcal{G}$ is called a query-response cycle. The following properties can be easily proved.

Property 1. If query-response pairs $Q$ and $Q^{\prime}$ are obtained by the first blockcipher call and the second blockcipher call, respectively, in an evaluation of $F$, then $\overrightarrow{Q Q^{\prime}} \in \mathcal{G}$. Conversely, each edge in $\mathcal{G}$ represents a valid evaluation of $F$.

Property 2. Each query-response cycle in $\mathcal{G}$ is of length 2 or length 6 . If $\Delta=\left(Q_{1}, \ldots, Q_{6}\right) \in \mathcal{G}$ is a cycle of length 6 , then we have

$$
\begin{array}{lll}
Q_{1}=\left(A_{1}, A_{2} \mid A_{3}, Y_{1}\right), & Q_{2}=\left(\overline{A_{2}}, A_{3} \mid A_{1}, Y_{2}\right), & Q_{3}=\left(\overline{A_{3}}, A_{1} \mid \overline{A_{2}}, Y_{3}\right), \\
Q_{4}=\left(\overline{A_{1}}, \overline{A_{2}} \mid \overline{A_{3}}, Y_{4}\right), & Q_{5}=\left(A_{2}, \overline{A_{3}} \mid \overline{A_{1}}, Y_{5}\right), & Q_{6}=\left(A_{3}, \overline{A_{1}} \mid A_{2}, Y_{6}\right),
\end{array}
$$

for some $A_{s}$ 's and $Y_{t}$ 's. If $\Delta=\left(Q_{1}, Q_{2}\right) \in \mathcal{G}$ is a cycle of length 2 , then we have $Q_{1}=$ $\left(A_{1}, A_{1} \mid \overline{A_{1}}, Y_{1}\right)$ and $Q_{2}=\left(\overline{A_{1}}, \overline{A_{1}} \mid A_{1}, Y_{2}\right)$ for some $A_{1}, Y_{1}$ and $Y_{2}$. Here we see that the first three blocks of the query-response pairs are moving cyclically under the permutation

$$
\begin{aligned}
\pi: I_{n}^{3} & \longrightarrow I_{n}^{3} \\
\left(A_{1}, A_{2}, A_{3}\right) & \longmapsto\left(\overline{A_{2}}, A_{3}, A_{1}\right) .
\end{aligned}
$$

Property 3. For query-response cycles $\Delta$ and $\Delta^{\prime}$, either $\Delta=\Delta^{\prime}$ or $\Delta \cap \Delta^{\prime}=\emptyset$.

Given an adversary $\mathcal{A}$ with oracle access to $E$ and $E^{-1}$, one can transform $\mathcal{A}$ into an adversary $\mathcal{B}$ that records its query history in terms of query-response cycles. The modified adversary $\mathcal{B}$ is described in Figure 3. We can easily check the following properties of $\mathcal{B}$.

Property 4. If $\mathcal{A}$ makes at most $q$ queries, then the corresponding adversary $\mathcal{B}$ makes at most $6 q$ queries, and records at most $q$ query-response cycles.

Property 5. $\mathbf{A d v}_{F}^{\mathrm{sec}}(\mathcal{A}) \leq \mathbf{A d v}_{F}^{\mathrm{sec}}(\mathcal{B})$ for sec $\in\{$ coll, pre $\}$.

\subsection{Security Results}

Given Property 5, we will analyze the security of the ABREAST-DM compression function with respect to the modified adversary $\mathcal{B}$. We denote the query history of $\mathcal{B}$ by

$$
\mathcal{Q}_{\Delta}=\left\{\Delta^{i}: 1 \leq i \leq q\right\},
$$

where we write $\Delta^{i}=\left(Q_{1}^{i}, Q_{2}^{i}, Q_{3}^{i}, Q_{4}^{i}, Q_{5}^{i}, Q_{6}^{i}\right)$ or $\left(Q_{1}^{i}, Q_{2}^{i}\right)$ for $1 \leq i \leq q$. Here we assume that query-response pair $Q_{j}^{i}$ is obtained after $Q_{j^{\prime}}^{i}$ if $j>j^{\prime}$.

Collision Resistance Let $\mathcal{E}$ denote the event that $\mathcal{B}$ makes a collision of $F$. Then, by definition, $\operatorname{Adv}_{F}^{\text {coll }}(\mathcal{B})=\operatorname{Pr}[\mathcal{E}]$. In order to estimate $\operatorname{Pr}[\mathcal{E}]$, we decompose $\mathcal{E}$ as follows.

$$
\mathcal{E}=\bigcup_{i=1}^{q}\left(\mathcal{E}^{i} \cup \bigcup_{j=1}^{i-1} \mathcal{E}^{i, j}\right)
$$

where

$$
\begin{aligned}
\mathcal{E}^{i} & \Leftrightarrow \text { two evaluations from a single cycle } \Delta^{i} \text { determines a collision, } \\
\mathcal{E}^{i, j} & \Leftrightarrow \text { two evaluations from } \Delta^{i} \text { and } \Delta^{j} \text { determine a collision. }
\end{aligned}
$$

Then it follows that

$$
\operatorname{Pr}[\mathcal{E}]=\sum_{i=1}^{q}\left(\operatorname{Pr}\left[\mathcal{E}^{i}\right]+\sum_{j=1}^{i-1} \operatorname{Pr}\left[\mathcal{E}^{i, j}\right]\right)
$$




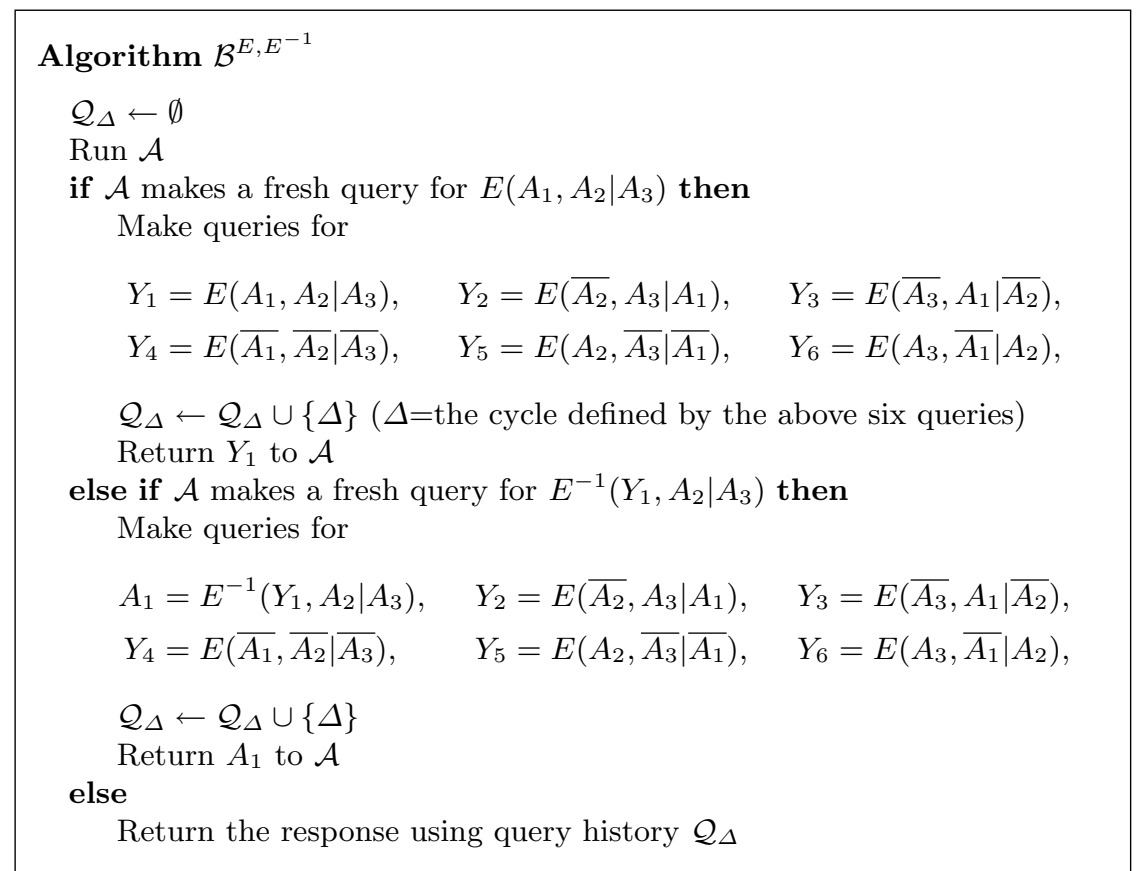

Fig. 3. Modified algorithm $\mathcal{B}$. A query is called "fresh" if its response is not obtained from the query history of $\mathcal{B}$

Lemma 1. Let $N^{\prime}=2^{n}-6 q \geq 15$ and $1 \leq i<j \leq q$. Then,

$$
\begin{aligned}
& \text { 1. } \operatorname{Pr}\left[\mathcal{E}^{i}\right] \leq 1 / N^{\prime}, \\
& \text { 2. } \operatorname{Pr}\left[\mathcal{E}^{i, j}\right] \leq 36 /\left(N^{\prime}\right)^{2} .
\end{aligned}
$$

Proof. Inequality 1: First, assume that $\Delta^{i}$ consists of two distinct query-response pairs. A collision within this cycle implies that $Q_{1}^{i}=\left(A_{1}, A_{1} \mid \overline{A_{1}}, Y_{1}\right), Q_{2}^{i}=\left(\overline{A_{1}}, \overline{A_{1}} \mid A_{1}, Y_{2}\right)$ and $\left(A_{1} \oplus Y_{1}, \overline{A_{1}} \oplus Y_{2}\right)=$ $\left(\overline{A_{1}} \oplus Y_{2}, A_{1} \oplus Y_{1}\right)$ for some $A_{1}, Y_{1}$ and $Y_{2}$. Since the second query-response pair $Q_{2}^{i}$ is obtained by a forward query, and $Y_{2}$ should be equal to $\overline{Y_{1}}$, the probability that this type of collision occurs is not greater than $1 / N^{\prime}$.

Next, assume that $\Delta^{i}$ consists of six distinct query-response pairs. Suppose that, say, $\overrightarrow{Q_{1}^{i} Q_{2}^{i}}$ and $\overrightarrow{Q_{2}^{i} Q_{3}^{i}}$ determines a collision. With the notations in Property 2, it should be the case that $\left(A_{1} \oplus Y_{1}, \overline{A_{2}} \oplus Y_{2}\right)=\left(\overline{A_{2}} \oplus Y_{2}, \overline{A_{3}} \oplus Y_{3}\right)$. In this case, we have $Y_{2}=A_{1} \oplus Y_{1} \oplus \overline{A_{2}}$ and $Y_{3}=\overline{A_{2}} \oplus Y_{2} \oplus \overline{A_{3}}$. The probability that $Y_{2}$ and $Y_{3}$ satisfy these equations is not greater than $\left(1 / N^{\prime}\right)^{2}$. The same argument applies to every pair of edges in $\Delta^{i}$. Since the number of such pairs is $\left(\begin{array}{l}6 \\ 2\end{array}\right)=15$ and $15 /\left(N^{\prime}\right)^{2} \leq 1 / N^{\prime}$ for $N^{\prime} \geq 15$, the first inequality is proved.

Inequality 2: Let $\overrightarrow{Q_{h}^{i} Q_{h+1}^{i}}$ and $\overrightarrow{Q_{h^{\prime}}^{j} Q_{h^{\prime}+1}^{j}}$ be edges contained in $\Delta^{i}$ and $\Delta^{j}$, respectively. Then we can write

$$
\begin{aligned}
Q_{h}^{i} & =\left(A_{1}, A_{2} \mid A_{3}, Y_{1}\right), & Q_{h+1}^{i} & =\left(\overline{A_{2}}, A_{3} \mid A_{1}, Y_{2}\right), \\
Q_{h^{\prime}}^{j} & =\left(A_{1}^{\prime}, A_{2}^{\prime} \mid A_{3}^{\prime}, Y_{1}^{\prime}\right), & Q_{h^{\prime}+1}^{j} & =\left(\overline{A_{2}^{\prime}}, A_{3}^{\prime} \mid A_{1}^{\prime}, Y_{2}^{\prime}\right),
\end{aligned}
$$

for some $A_{s}$ 's, $A_{s}^{\prime}$ 's, $Y_{t}$ 's and $Y_{t}^{\prime}$ 's. If two edges $\overrightarrow{Q_{h}^{i} Q_{h+1}^{i}}$ and $\overrightarrow{Q_{h^{\prime}}^{j} Q_{h^{\prime}+1}^{j}}$ determine a collision, then it should be the case that $\left(A_{1} \oplus Y_{1}, \overline{A_{2}} \oplus Y_{2}\right)=\left(A_{1}^{\prime} \oplus Y_{1}^{\prime}, \overline{A_{2}^{\prime}} \oplus Y_{2}^{\prime}\right)$, or equivalently $Y_{1}^{\prime}=A_{1} \oplus Y_{1} \oplus A_{1}^{\prime}$ and $Y_{2}^{\prime}=\overline{A_{2}} \oplus Y_{2} \oplus \overline{A_{2}^{\prime}}$. The probability that such an event occurs is not greater than $\left(1 / N^{\prime}\right)^{2}$. Since each cycle contains at most 6 edges, we obtain $\operatorname{Pr}\left[\mathcal{E}^{i, j}\right] \leq 36 /\left(N^{\prime}\right)^{2}$. 
By Lemma 1, equality (10) and Property 5, we obtain the following theorem.

Theorem 1. Let $F^{A B R}$ be the compression function ABREAST-DM and let $q>0$. Then,

$$
\mathbf{A d v}_{F^{A B R}}^{\text {coll }}(q) \leq \frac{q}{\left(2^{n}-6 q\right)}+\frac{18 q^{2}}{\left(2^{n}-6 q\right)^{2}}
$$

Preimage Resistance Suppose that a modified adversary $\mathcal{B}$ is given a target image $B=\left(B_{1}, B_{2}\right)$. Let $\mathcal{E}$ denote the event that $\mathcal{B}$ makes an evaluation $F\left(A_{1}, A_{2}, A_{3}\right)=\left(B_{1}, B_{2}\right)$ for some $A_{s}$ 's. Then, by definition, $\operatorname{Adv}_{F}^{\text {pre }}(\mathcal{B})=\operatorname{Pr}[\mathcal{E}]$. Define

$$
\mathcal{E}^{i} \Leftrightarrow \Delta^{i} \text { determines a preimage of } B \text {. }
$$

Then it follows that

$$
\operatorname{Pr}[\mathcal{E}]=\sum_{i=1}^{q} \operatorname{Pr}\left[\mathcal{E}^{i}\right]
$$

Let $\overrightarrow{Q_{h}^{i} Q_{h+1}^{i}}$ be an edge contained in $\Delta^{i}$. Then we can write $Q_{h}^{i}=\left(A_{1}, A_{2} \mid A_{3}, Y_{1}\right)$ and $Q_{h+1}^{i}=$ $\left(\overline{A_{2}}, A_{3} \mid A_{1}, Y_{2}\right)$ for some $A_{s}$ 's and $Y_{t}$ 's. If $\overrightarrow{Q_{h}^{i} Q_{h+1}^{i}}$ determines a preimage of $B=\left(B_{1}, B_{2}\right)$, then it should be the case that $\left(A_{1} \oplus Y_{1}, \overline{A_{2}} \oplus Y_{2}\right)=\left(B_{1}, B_{2}\right)$, or equivalently $Y_{1}=B_{1} \oplus A_{1}$ and $Y_{2}=B_{2} \oplus \overline{A_{2}}$. The probability that such an event occurs is not greater than $\left(1 / N^{\prime}\right)^{2}$. Since each cycle contains at most 6 edges, we obtain $\operatorname{Pr}\left[\mathcal{E}^{i}\right] \leq 6 /\left(N^{\prime}\right)^{2}$ for $1 \leq i \leq q$, and the following theorem.

Theorem 2. Let $F^{A B R}$ be the compression function ABREAST-DM and let $q>0$. Then,

$$
\operatorname{Adv}_{F^{A B R}}^{\text {pre }}(q) \leq \frac{6 q}{\left(2^{n}-6 q\right)^{2}}
$$

\section{Abreast-DM Variants}

In this section, we present a wide class of ABREAST-DM variants that enjoy a birthday-type security guarantee. Let $\pi$ be a permutation on $I_{n}^{3}\left(\equiv I_{n} \times I_{n}^{2}\right)$ such that every cycle in $\pi$ is of length $2 \leq l \leq L$ for a positive integer $L$. Then we can associate the permutation $\pi$ with an ABREAST-DM variant $F_{\pi}^{A B R}$ as follows.

$$
\begin{aligned}
F_{\pi}^{A B R}: I_{n}^{3} & \longrightarrow I_{n}^{2} \\
\left(A_{1}, A_{2}, A_{3}\right) & \longmapsto\left(E\left(X_{1}, K_{1}\right) \oplus X_{1}, E\left(X_{2}, K_{2}\right) \oplus X_{2}\right),
\end{aligned}
$$

where $\left(X_{1}, K_{1}\right)=\left(A_{1}, A_{2} \mid A_{3}\right)$ and $\left(X_{2}, K_{2}\right)=\pi\left(A_{1}, A_{2}, A_{3}\right)$. An ABreAst-DM variant is illustrated in Figure 4. By essentially the same argument as the previous section, we can prove the following theorem.

Theorem 3. Let $F_{\pi}^{A B R}$ be the compression function defined in (13), and let $2^{n} \geq q+\left(\begin{array}{l}L \\ 2\end{array}\right)$. Then,

$$
\begin{aligned}
& \operatorname{Adv}_{F_{\pi}^{A B R}}^{\text {coll }}(q) \leq \frac{q}{\left(2^{n}-L q\right)}+\frac{L^{2} q^{2}}{2\left(2^{n}-L q\right)^{2}}, \\
& \mathbf{A d v}_{F_{\pi}^{A B R}}^{\text {pre }}(q) \leq \frac{L q}{\left(2^{n}-L q\right)^{2}}
\end{aligned}
$$

If $\pi$ contains no cycle of length 2 , then

$$
\operatorname{Adv}_{F_{\pi}^{A B R}}^{\text {coll }}(q) \leq \frac{L^{2}\left(q+q^{2}\right)}{2\left(2^{n}-L q\right)^{2}}
$$




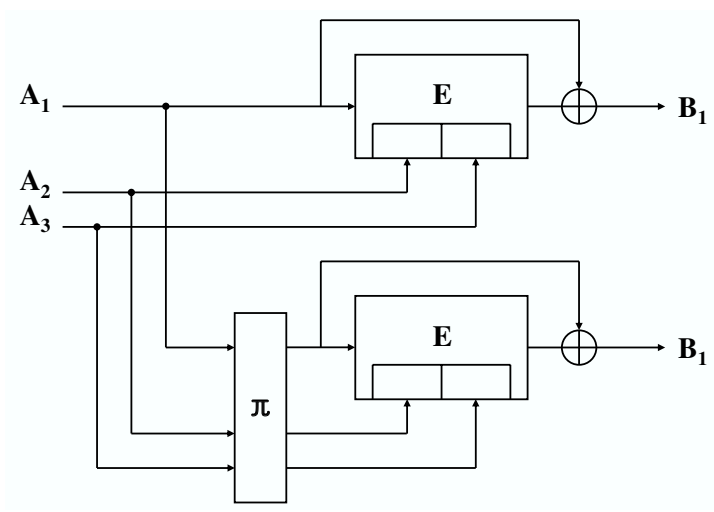

Fig. 4. Abreast-DM variant

We conclude this section with some examples.

Example 1. Let $\pi:\left(A_{1}, A_{2}, A_{3}\right) \mapsto\left(A_{1} \oplus C, A_{2}, A_{3}\right)$ for a constant $C \in I_{n}$. Then $F_{\pi}^{A B R}$ is reduced to the Hirose's scheme [11].

Example 2. Let $\pi:\left(A_{1}, A_{2}, A_{3}\right) \mapsto\left(\overline{A_{1}}, A_{3}, \overline{A_{2}}\right)$. Then every cycle in $\pi$ is of length 4 . By Theorem 3, we have

$$
\operatorname{Adv}_{F_{\pi}^{A B R}}^{\text {coll }}(q) \leq \frac{8\left(q+q^{2}\right)}{\left(2^{n}-4 q\right)^{2}}
$$

In numerical terms with $n=128$, any adversary asking less than $2^{125.0}$ queries cannot find a collision with probability greater than $1 / 2$.

\section{Conclusion}

In this paper, we analyzed collision resistance and preimage resistance of ABREAST-DM with a novel technique using query-response cycles. As a result, we have shown that ABREAST-DM is both collision resistant and preimage resistant up to $O\left(2^{n}\right)$ query complexity. With essentially the same proof as ABREAST-DM, we also presented a wide class of ABREAST-DM variants that enjoy a birthday-type security guarantee. We note that, however, our result for preimage resistance might not be optimal, since a truly random function with a $2 n$-bit output would require $O\left(2^{2 n}\right)$ queries to find any preimage. For this reason, it will be an interesting further research to improve the security proof for premiage resistance.

\section{References}

1. E. Biham, R. Chen, A. Joux, P. Carribault, C. Lemuet and W. Jalby. Collisions of SHA-0 and reduced SHA-1. Eurocrypt 2005, LNCS 3494, pp. 36-57, Springer-Verlag, 2005.

2. J. Black, M. Cochran and T. Shrimpton. On the impossibility of highly-efficient blockcipher-based hash functions. Eurocrypt 2005, LNCS 3494, pp. 526-541, Springer-Verlag, 2005.

3. A. Bogdanov, G. Leander, C. Paar, A. Poschmann, M. J. B. Robshaw and Y. Seurin. Hash functions and RFID tags: mind the gap. CHES 2008, LNCS 5154, pp. 283-299, Springer-Verlag, 2008.

4. J. Black, P. Rogaway and T. Shrimpton. Black-box analysis of the block-cipher-based hash-function construction from PGV. Crypto 2002, LNCS 2442, pp. 320-325, Springer-Verlag, 2002.

5. B. Brachtl, D. Coppersmith, M. Heyden, S. Matyas, C. Meyer, J. Oseas, S. Pilpel and M. Schilling. Data authentication using modification detection codes based on a public one-way encryption function. US Patent \#4,908,861. Awarded March 13, 1990 (filed August 28, 1987). 
6. De. Canniere and C. Rechberger. Preimages for reduced SHA-0 and SHA-1. Crypto 2008, LNCS 5157, pp. 179-202, Springer-Verlag, 2008.

7. E. Fleischmann, M. Gorski and S. Lucks. On the security of TANDEM-DM. Preproceedings of FSE 2009, pp. 85-105, 2009.

8. M. Hattori, S. Hirose and S. Yoshida. Analysis of double block length hash functions. IMA 2003, LNCS 2898, pp. 290-302, Springer-Verlag, 2003.

9. S. Hirose. A security analysis of double-block-length hash functions with the rate 1. IEICE Transactions on Fundamentals of Electronics, Communications and Computer Sciences, Vol. E89-A, NO. 10, pp. 25752582, 2006.

10. S. Hirose. Provably secure double-block-length hash functions in a black-box model. ICISC 2004, LNCS 3506, pp. 330-342, Springer-Verlag, 2005.

11. S. Hirose. Some plausible construction of double-block-length hash functions. FSE 2006, LNCS 4047, pp. 210-225, Springer-Verlag, 2006.

12. L. R. Knudsen, J. L. Massey and B. Preneel. Attacks on fast double block length hash functions. Journal of Cryptology, Vol. 11, NO. 1, pp. 59-72, 1998.

13. X. Lai and J. L. Massey. Hash function based on block ciphers. Eurocrypt 1992, LNCS 658, pp. 55-70, Springer-Verlag, 1993.

14. G. Leurent. MD4 is not one-way. FSE 2008, LNCS 5086, pp. 412-428, Springer-Verlag, 2008.

15. F. Mendel, N. Pramstaller, C. Rechberger and V. Rijmen. Analysis of step-reduced SHA-256. FSE 2006, LNCS 4047, pp. 126-143, Springer-Verlag, 2006.

16. B. Preneel, R. Govaerts and J. Vandewalle. Hash functions based on block ciphers: A synthetic approach. Crypto 1993, LNCS 773, pp. 368-378, Springer-Verlag, 1994.

17. T. Ristenpart and T. Shrimpton. How to build a hash function from any collision-resistant function. Asiacrypt 2007, LNCS 4833, pp. 147-163, Springer-Verlag, 2007.

18. P. Rogaway and J. Steinberger. Constructing cryptographic hash functions from fixed-key blockciphers. Crypto 2008, LNCS 5157, pp. 433-450, Springer-Verlag, 2008.

19. P. Rogaway and J. Steinberger. Security/efficiency tradeoffs for permuation-based hashing. Eurocrypt 2008, LNCS 4965, pp. 220-236, Springer-Verlag, 2008.

20. Y. Sasaki and K. Aoki. Finding preimages in full MD5 faster than exhaustive search. Eurocrypt 2009, LNCS 5479, pp. 134-152, Springer-Verlag, 2008.

21. T. Shrimpton and M. Stam. Building a collision-resistant function from non-compressing primitives. ICALP 2008, LNCS 5126, pp. 643-654, Springer-Verlag, 2008.

22. M. Stam. Beyond uniformity: Better security/efficiency tradeoffs for compression functions. Crypto 2008, LNCS 5157, pp. 397-412, Springer-Verlag, 2008.

23. J. Steinberger. The collision intractability of MDC-2 in the ideal-cipher model. Eurocrypt 2007, LNCS 4515, pp. 34-51, Springer-Verlag, 2008.

24. X. Wang, X. Lai, D. Feng, H. Chen and X. Yu. Cryptanalysis of the hash functions MD4 and RIPEMD. Eurocrypt 2005, LNCS 3494, pp. 1-18, Springer-Verlag, 2005.

25. X. Wang, X. Lai and H. Yu. Finding collisions in the full SHA-1. Crypt0 2005, LNCS 3621, pp. 17-36, Springer-Verlag, 2005.

26. X. Wang and H. Yu. How to break MD5 and other hash functions. Eurocrypt 2005, LNCS 3494, pp. 19-35, Springer-Verlag, 2005. 